Int. J. Electrochem. Sci., 13 (2018) $7129-7140$

\title{
A simple and Novel Electroanalytical Method for Determination of Brain Serotonin Based on the MWNTs/ $/ \mathrm{Al}_{2} \mathrm{O}_{3} /$ chitosan SPE
}

\author{
Weigang Wu ${ }^{1, \#}$, Caiyan Liu $^{2, \#}$, Fang Huang ${ }^{3, \#}$, Huaying Li $^{1}$, Yuting Wang ${ }^{5}$,Ding Hong ${ }^{5}$, Shi Wang ${ }^{*}$, \\ Xiaobin Zeng ${ }^{1, *}$ \\ ${ }^{1}$ Center Lab of Longhua Branch, Shenzhen People's Hospital, 2nd Clinical Medical College of Jinan \\ University, Shenzhen 518120, Guangdong Province, China. \\ ${ }^{2}$ Department of Clinical Laboratory, Shenzhen People's Hospital, 2nd Clinical Medical College of \\ Jinan University, Shenzhen 518120, Guangdong Province, China. \\ ${ }^{3}$ Shenzhen Third People's Hospital, The Second Affiliated Hospital of Shenzhen University, Shenzhen \\ 518120, Guangdong Province, China. \\ ${ }^{4}$ School of Pharmacy, Hubei University of Science and Technology, Xianning, 437100, China \\ ${ }^{5}$ Department of Pharmacy, Wuhan University, Wuhan 430072, China \\ \# These authors equally contributed to this work. \\ *E-mail: 912692441@qq.com, zengxiaobin1983@163.com
}

doi: $10.20964 / 2018.07 .69$

Received: 13 April 2018 / Accepted: 22 May 2018 / Published: 5 June 2018

In this work, a simple and novel method for determination of brain serotonin (5-HT) in depression mice was proposed with modified screen-printed electrode based on $\mathrm{MWNTs} / \mathrm{Al}_{2} \mathrm{O}_{3} /$ chitosan. The modified SPE displayed excellent electrocatalytic activity towards the oxidation of 5-HT in real brain tissue samples. Square wave voltammetry (SWV) was employed to optimize the experimental conditions. The proposed sensor showed voltammetric responses with high and good sensitivity for 5HT in optimal conditions. The peak current of 5-HT was proportional to the concentration in the range of $0.01 \mu \mathrm{M}$ to $1.0 \mu \mathrm{M}$ with the detection limit $0.005 \mu \mathrm{M}$. Compared with the HPLC method, the newly developed method also presented good results in the detection of brain 5-HT level, which made it possible for helping depression desiease diagnosis. In conlusion, our experimental results strongly suggested that the established SPE could be used to the fast depression determination of 5-HT, which might offer a potential minotor tool for in future.

Keywords: Disposable sensor, brain serotonin, modified screen printed electrode, clinical depression diagnosis 
(C) 2018 The Authors. Published by ESG (www.electrochemsci.org). This article is an open access article distributed under the terms and conditions of the Creative Commons Attribution license (http://creativecommons.org/licenses/by/4.0/). 